\title{
Nonexistence of Chebyshev-Type Quadratures on Infinite Intervals*
}

\author{
By Walter Gautschi
}

Dedicated to D. H. Lehmer on his 70 th birthday

\begin{abstract}
Quadrature rules on semi-infinite and infinite intervals are considered involving weight functions of the Laguerre and Hermite type. It is shown that such quadrature rules cannot have equal coefficients and real nodes unless the algebraic degree of accuracy is severely limited.
\end{abstract}

1. Introduction. Given a nonnegative weight function $w(x)$ on the interval $(a, b)$, with finite moments

$$
m_{k}=\int_{a}^{b} x^{k} w(x) d x, \quad k=0,1,2, \cdots, \quad m_{0}>0
$$

a quadrature rule of the form

$$
\int_{a}^{b} f(x) w(x) d x=\frac{m_{0}}{n} \sum_{k=1}^{n} f\left(x_{k}^{(n)}\right)+R_{n}(f)
$$

is called a Chebyshev quadrature formula if the nodes $x_{k}^{(n)}$ are mutually distinct and located in $(a, b)$, and if (1.1) has polynomial degree of accuracy $n$, i.e., $R_{n}(f)=0$ whenever $f$ is a polynomial of degree $\leqslant n$. In the following we are going to relax these requirements in two respects: Firstly, we shall drop the requirement that the nodes be distinct, or even contained in $(a, b)$, assuming merely that all be real. Secondly, we shall allow for polynomial degree of accuracy $<n$. Since we are interested in questions of nonexistence, both modifications only strengthen our results. We shall refer to quadrature rules (1.1), under these relaxed conditions, as Chebyshev-type quadrature formulas.

We focus our attention on two special cases of (1.1): the Chebyshev-Laguerre formula

$$
\int_{0}^{\infty} x^{\alpha} e^{-x} f(x) d x=\frac{\Gamma(\alpha+1)}{n} \sum_{k=1}^{n} f\left(x_{k}^{(n)}\right)+R_{n}(f), \quad \alpha>-1
$$

Received February 27, 1974.

AMS (MOS) subject classifications (1970). Primary 65D30.

${ }^{*}$ This work was supported in part by the National Science Foundation under grant GP-36557. 
and the Chebyshev-Hermite formula

$$
\int_{-\infty}^{\infty} e^{-x^{2}} f(x) d x=\frac{\sqrt{\pi}}{n} \sum_{k=1}^{n} f\left(x_{k}^{(n)}\right)+R_{n}(f) .
$$

In 1955, H. E. Salzer [5] first observed (by computation) that a Chebyshev formula (1.2), in the strict sense, for $\alpha=0$, exists for $n=1$ and $n=2$, but does not exist for $3 \leqslant n \leqslant 10$. He found similarly that (1.3) exists for $n=1,2$ and 3 , but not for $4 \leqslant n \leqslant 10$. Shortly thereafter, V. I. Krylov [4] proved that in fact (1.2) (with $\alpha=0$ ) and (1.3) do not exist for any $n \geqslant 3$ and $n \geqslant 4$, respectively. This was proved again later on, independently, by H. S. Wilf [6] and L. Gatteschi [3]. We shall prove below analogous results for Chebyshev-type quadratures. In particular, we show that formulas (1.2) and (1.3) with polynomial degrees of accuracy $p_{n} \geqslant \tau n^{\gamma}, \tau>0,1 / 2<\gamma<1$, as $n \rightarrow \infty$, cannot exist.

Both Krylov's and Gatteschi's proofs are based on an inequality of Bernstein, which was used by Bernstein to settle the existence of Chebyshev quadratures in the classical case $w(x)=1$ on $[-1,1]$. We shall continue using Bernstein's method, but simplify its application and also establish its validity in the case of nodes not necessarily distinct.

2. Bernstein's Inequalities. The lemmas in this section are slight extensions or modifications of results due to S. Bernstein [2], which in turn, according to Bernstein [loc. cit., p. 180], are "but more or less immediate applications or modifications of a proposition due to Chebyshev". Our extension is to arbitrary weight functions (which is immediate), and our modification is prompted by our reluctance to assume mutually distinct nodes.

Let $\xi_{r}^{(m)}$ and $\lambda_{r}^{(m)}$ be the abscissas and weights of the $m$-point Gaussian quadrature rule

$$
\int_{a}^{b} f(x) w(x) d x=\sum_{r=1}^{m} \lambda_{r}^{(m)} f\left(\xi_{r}^{(m)}\right)+R_{m}(f), \quad \xi_{m}^{(m)}<\xi_{m-1}^{(m)}<\cdots<\xi_{1}^{(m)}
$$

Thus, $R_{m}(f)=0$ for all polynomials of degree $\leqslant 2 m-1$. The abscissas $\xi_{r}^{(m)}$ are the zeros of the $m$ th degree orthogonal polynomial associated with the weight function $w(x)$, while the weights $\lambda_{r}^{(m)}$ are the corresponding Christoffel numbers.

LEMmA 2.1. If the Chebyshev-type quadrature formula (1.1) has polynomial degree of accuracy $2 m-1, m<n$, then either

$$
x_{r}^{(n)}=\xi_{r}^{(m)}\left(n_{r} \text {-times }\right), \quad \lambda_{r}^{(m)}=\left(m_{0} / n\right) n_{r}, \quad r=1,2, \cdots, m,
$$

where $n_{r} \geqslant 1$ are integers summing up to $n$, or

$$
x_{k}^{(n)}>\xi_{1}^{(m)}
$$

for at least one value of $k, 1 \leqslant k \leqslant n$. The same statement holds true with (2.3) replaced by 


$$
x_{k}^{(n)}<\xi_{m}^{(m)}
$$

The proof utilizes the polynomial $p(x)=p_{m}^{2}(x) /\left(x-\xi_{1}^{(m)}\right)$, where $p_{m}$ is the $m$ th degree orthogonal polynomial associated with the weight function $w(x)$. We have, on the one hand $\int_{a}^{b} p(x) w(x) d x=0$, and on the other, by assumption,

$$
\sum_{k=1}^{n} p\left(x_{k}^{(n)}\right)=0
$$

There are the following alternatives:

(i) All $x_{k}^{(n)} \leqslant \xi_{1}^{(m)}$. Since $p(x) \leqslant 0$ for $x \leqslant \xi_{1}^{(m)}$, and $p(x)=0$ only for $x=\xi_{r}^{(m)}, r=1,2, \cdots, m$, it follows from (2.4) that all $x_{k}^{(n)}$ must be placed at Gaussian nodes $\xi_{r}^{(m)}$, that is,

$$
x_{r}^{(n)}=\xi_{r}^{(m)} \quad\left(n_{r} \text {-times), } \quad r=1,2, \cdots, m,\right.
$$

where $n_{r} \geqslant 0$ are integers with $n_{1}+n_{2}+\cdots+n_{m}=n$. The quadrature rule (1.1) then becomes

$$
\int_{a}^{b} f(x) w(x) d x=\frac{m_{0}}{n} \sum_{r=1}^{m} n_{r} f\left(\xi_{r}^{(m)}\right)+R_{n}(f) .
$$

Since it has polynomial degree of accuracy $2 m-1$, and since the Gaussian quadrature rule (2.1) is unique, we conclude (2.2), and in particular, $n_{r} \geqslant 1$ for all $r$, by virtue of $\lambda_{r}^{(m)}>0$. This proves the first alternative (2.2).

(ii) At least one $x_{k}^{(n)}>\xi_{1}^{(m)}$. This gives the second alternative (2.3).

The statement concerning (2.2) and (2.3') follows similarly by using $p(x)=$ $p_{m}^{2}(x) /\left(x-\xi_{m}^{(m)}\right)$.

Lemma 2.2. If the Chebyshev-type quadrature formula (1.1) has polynomial degree of accuracy $2 m-2$, then

$$
\sigma\left(\xi_{1}^{(m)}\right) m_{0} / n \leqslant \lambda_{1}^{(m)} \text { and } \tau\left(\xi_{m}^{(m)}\right) m_{0} / n \leqslant \lambda_{m}^{(m)},
$$

where $\sigma\left(\xi_{1}^{(m)}\right)$ and $\tau\left(\xi_{m}^{(m)}\right)$ are the number of nodes $x_{k}^{(n)}$ with $x_{k}^{(n)} \geqslant \xi_{1}^{(m)}$ and $x_{k}^{(n)} \leqslant \xi_{m}^{(m)}$, respectively.

The proof of Bernstein, utilizing $p(x)=p_{m}^{2}(x)\left(x-\xi_{r}^{(m)}\right)^{-2}\left[p_{m}^{\prime}\left(\xi_{r}^{(m)}\right)\right]^{-2}$, $r=1$ or $r=m$, applies essentially unchanged.

LEMma 2.3. If the Chebyshev-type quadrature formula (1.1) has polynomial degree of accuracy $2 m-1, m<n$, then

$$
m_{0} / n \leqslant \min \left\{\lambda_{1}^{(m)}, \lambda_{m}^{(m)}\right\}
$$

Proof. By Lemma 2.1 we have either (2.2), or else both (2.3) and (2.3'). In the first case, (2.6) results from putting $r=1$ and $r=m$ in (2.2) and noting that $n_{r} \geqslant 1$. In the second case, $(2.6)$ is a consequence of $(2.5)$.

3. Nonexistence of Chebyshev-Laguerre Type Formulas.

Theorem 3.1. A Chebyshev-type quadrature formula (1.2) having polynomial 
degree of accuracy $n-s, s \geqslant 0$, does not exist if

$$
n \geqslant s+1 / 2\{\alpha+5+\sqrt{(\alpha+1)(\alpha+5+4 s)}\} \text { and } n-s \text { (even) } \geqslant 4,
$$

or if

$$
n \geqslant s-1+1 / 2\{\alpha+5+\sqrt{(\alpha+1)(\alpha+1+4 s)}\} \text { and } n-s(\text { odd }) \geqslant 3 .
$$

Remark. For $n-s=2$ it is easily shown that Chebyshev-type formulas (1.2) do indeed exist. The same, of course, is true if $n-s=1$.

Proof of Theorem 3.1. Let $\pi_{0}(x), \pi_{1}(x), \cdots$ denote the normalized Laguerre polynomials $L_{0}^{(\alpha)}(x), L_{1}^{(\alpha)}(x), \cdots$, and $\xi_{r}^{(m)}$ the zeros of $\pi_{m}$, ordered decreasingly. The associated Christoffel numbers are

$$
\lambda_{r}^{(m)}=1 / \sum_{k=0}^{m-1}\left[\pi_{k}\left(\xi_{r}^{(m)}\right)\right]^{2} .
$$

V. I. Krylov [4] showed that

$$
\xi_{1}^{(m)}>2 m+\alpha-1 \quad(m \geqslant 2, \alpha>-1) .
$$

Since

$$
\pi_{0}=[\Gamma(\alpha+1)]^{-1 / 2}, \quad \pi_{1}(x)=[\Gamma(\alpha+2)]^{-1 / 2}(\alpha+1-x),
$$

it follows from (3.3) that

$$
\lambda_{1}^{(m)} \leqslant \frac{1}{\pi_{0}^{2}+\left[\pi_{1}\left(\xi_{1}^{(m)}\right)\right]^{2}}=\frac{\Gamma(\alpha+1)}{1+\left[\xi_{1}^{(m)}-(\alpha+1)\right]^{2} /(\alpha+1)},
$$

and thus from (3.4) that

$$
\lambda_{1}^{(m)}<\frac{\Gamma(\alpha+1)}{1+4(m-1)^{2} /(\alpha+1)}, \quad m \geqslant 2 .
$$

We first consider the case where $n-s$ is even. Let $n-s=2 m, m \geqslant 2$. By Bernstein's inequality (2.6) we have nonexistence of (1.2) if $\Gamma(\alpha+1) / n>$ $\lambda_{1}^{(m)}$, hence, a fortiori, if

$$
\frac{1}{n} \geqslant \frac{1}{1+4(m-1)^{2} /(\alpha+1)}, \quad m \geqslant 2 .
$$

Since $n=2 m+s$, the last inequality is equivalent to

$$
4 m^{2}-(2 \alpha+10) m+\alpha+5-s(\alpha+1) \geqslant 0,
$$

and is satisfied if

$$
m \geqslant 1 / 4\{\alpha+5+\sqrt{(\alpha+1)(\alpha+5+4 s)}\}, \quad m \geqslant 2 .
$$

Since $m=(n-s) / 2$, this proves (3.1). 
It remains to consider the case $n-s$ odd, $n-s=2 m-1, m \geqslant 2$. Nonexistence of (1.2) now results if (3.5) holds with $n=2 m+s-1$, i.e., if

$$
4 m^{2}-(2 \alpha+10) m+2 \alpha+6-s(\alpha+1) \geqslant 0, \quad m \geqslant 2,
$$

or, in particular, if

$$
m \geqslant 1 / 4\{\alpha+5+\sqrt{(\alpha+1)(\alpha+1+4 s)}\}, \quad m \geqslant 2 .
$$

Since $m=(n-s+1) / 2$, this gives (3.2), and the proof of Theorem 3.1 is completed.

Corollary. There is no sequence $\left\{C_{n}\right\}$ of Chebyshev-type formulas (1.2), corresponding to a sequence $S$ of integers $n=n_{j}, n_{j} \rightarrow \infty$, such that $C_{n}$ has polynomial degree of accuracy $p_{n} \geqslant \tau n^{\gamma}, \tau>0,1 / 2<\gamma<1$, for each $n \in S$.

Proof. Letting $p_{n}=n-s_{n}$, we have $s_{n} \leqslant n-\tau n^{\gamma}$, and (3.1), (3.2) are both satisfied for $s=s_{n}$ and $n$ sufficiently large.

We remark that (3.1) and (3.2) in the case $\alpha=0$ are sharp for $s=0$ and $s=1$, giving the correct bounds $n \geqslant 3$ and $n \geqslant 4$, respectively, but are not quite sharp for $s \geqslant 2$. If $s=2$, the inequalities (3.1), (3.2) yield $n=5$ or $n \geqslant 7$. In reality, $n \geqslant 5$, as can be seen from Bernstein's inequality by verifying $n^{-1}>\lambda_{1}^{(2)}$ for $n=6$. For $s=3$ we obtain $n \geqslant 8$, while in reality $n \geqslant 6$. The latter follows from Bernstein's inequality when $n=6$, and from computations performed in [1] when $n=7$.

4. Nonexistence of Chebyshev-Hermite Type Formulas. We call (1.3) a symmetric Chebyshev-type quadrature formula if the nodes $x_{k}^{(n)}$ are located symmetrically with respect to the origin. Symmetric formulas are trivially exact for odd functions. We can assume therefore that the polynomial degree of accuracy of a symmetric formula is odd.

Theorem 4.1. A symmetric Chebyshev-type quadrature formula (1.3) having polynomial degree of accuracy $2[n / 2]-2 s+1, s \geqslant 0$, does not exist if

$$
s=0 \text { and } n \geqslant 4, s=1 \text { and } n \geqslant 8,
$$

$$
s>1 \text { and } n \geqslant \begin{cases}2(s+1+\sqrt{s}), & n \text { even, } \\ 2 s+3+\sqrt{4 s+2}, & n \text { odd } .\end{cases}
$$

Proof. The normalized Hermite polynomials $\pi_{0}(x), \pi_{1}(x), \cdots$ can be expressed in terms of Laguerre polynomials by means of

$$
\begin{aligned}
& \pi_{n}(x)=\left\{\frac{\Gamma(n / 2+1)}{\Gamma((n+1) / 2)}\right\}^{1 / 2} L_{n / 2}^{(-1 / 2)}\left(x^{2}\right), \quad n \text { even } \\
& \pi_{n}(x)=\left\{\frac{\Gamma((n+1) / 2)}{\Gamma(n / 2+1)}\right\}^{1 / 2} x L_{(n-1) / 2}^{(1 / 2)}\left(x^{2}\right), \quad n \text { odd }
\end{aligned}
$$


The largest zero $\xi_{1}^{(m)}$ of $\pi_{m}$, by (3.4) and (4.2), thus satisfies

$$
\begin{array}{ll}
{\left[\xi_{1}^{(m)}\right]^{2}>m-3 / 2} & \text { for } m \geqslant 4, \\
{\left[\xi_{1}^{(m)}\right]^{2}=m-3 / 2} & \text { for } m=2 \text { and } 3 .
\end{array}
$$

Since

$$
\pi_{0}=\pi^{-1 / 4}, \quad \pi_{1}(x)=2^{1 / 2} \pi^{-1 / 4} x, \quad \pi_{2}(x)=2^{-1 / 2} \pi^{-1 / 4}\left(2 x^{2}-1\right),
$$

we obtain for the corresponding Christoffel number, using again (3.3),

$$
\lambda_{1}^{(m)} \leqslant \frac{1}{\pi_{0}^{2}+\left[\pi_{1}\left(\xi_{1}^{(m)}\right)\right]^{2}+\left[\pi_{2}\left(\xi_{1}^{(m)}\right)\right]^{2}}=\frac{2 \sqrt{\pi}}{2+4\left[\xi_{1}^{(m)}\right]^{2}+\left(2\left[\xi_{1}^{(m)}\right]^{2}-1\right)^{2}},
$$

and thus, from (4.3),

$$
\begin{array}{ll}
\lambda_{1}^{(m)}<\frac{\sqrt{\pi}}{2\left[m-1+(m-2)^{2}\right]}, & m \geqslant 4, \\
\lambda_{1}^{(m)}=\frac{\sqrt{\pi}}{2\left[m-1+(m-2)^{2}\right]}, & m=2 \text { and } 3 .
\end{array}
$$

We first consider the case when $n$ is even, $n=2 v$. Since we are assuming polynomial degree of accuracy $2[n / 2]-2 s+1=2 v-2 s+1$, we have $m=v-s+1$ in Bernstein's inequality (2.6). Nonexistence of (1.3) thus follows in either of the following two cases:

$$
\begin{aligned}
& \frac{1}{2 \nu} \geqslant \frac{1}{2\left[\nu-s+(\nu-s-1)^{2}\right]} \text { and } v \geqslant s+3 \\
& \frac{1}{2 \nu}>\frac{1}{2\left[\nu-s+(\nu-s-1)^{2}\right]} \text { and } v=s+1 \text { or } s+2 .
\end{aligned}
$$

The first case is equivalent to

$$
v^{2}-2(s+1) v+s^{2}+s+1 \geqslant 0 \text { and } v \geqslant s+3,
$$

and is realized if

$$
n \geqslant 2(s+1+\sqrt{s}), \quad n(\text { even }) \geqslant 2 s+6 .
$$

The second case occurs if

$$
n>2(s+1+\sqrt{s}), \quad n=2 s+2 \text { or } 2 s+4 .
$$

Now $n=2 s+2$ in (4.5) implies $0>2 \sqrt{s}$, which is impossible, while $n=2 s+4$ implies $s=0$, hence $n=4$. Putting in turn $s=0$ and $s=1$ in (4.4) gives $n$ (even) $\geqslant 6$ and $n$ (even) $\geqslant 8$, respectively, while for $s \geqslant 2$ we get $n($ even $) \geqslant 2(s+1+\sqrt{s})$. 
Since in all these cases a symmetric formula (1.3) does not exist, Theorem 4.1 is proved for all even $n \geqslant 4$.

Consider now the case of odd $n, n=2 v+1$. The degree of accuracy still being $2 v-2 s+1$, we can again use $m=v-s+1$ in Bernstein's inequality and infer nonexistence of (1.3) in either of the following two cases:

$$
\begin{gathered}
\frac{1}{2 v+1} \geqslant \frac{1}{2\left[v-s+(\nu-s-1)^{2}\right]} \text { and } v \geqslant s+3, \\
\frac{1}{2 v+1}>\frac{1}{2\left[\nu-s+(\nu-s-1)^{2}\right]} \text { and } v=s+1 \text { or } s+2 .
\end{gathered}
$$

As before one sees that these cases hold if

$$
n \geqslant 2 s+3+\sqrt{4 s+2}, \quad n(\text { odd }) \geqslant 2 s+7
$$

and

$$
n>2 s+3+\sqrt{4 s+2}, \quad n=2 s+3 \text { or } 2 s+5,
$$

respectively. The latter is possible only if $s=0$, giving $n=5$. Putting $s=0$ in the former gives $n$ (odd) $\geqslant 7$, while for $s \geqslant 1$ we get $n$ (odd) $\geqslant 2 s+3+\sqrt{4 s+2}$. This proves Theorem 4.1 for all odd $n \geqslant 5$.

COROLlARY. There is no sequence $\left\{C_{n}\right\}$ of symmetric Chebyshev-type formulas (1.3), corresponding to a sequence $S$ of integers $n=n_{j}, n_{j} \rightarrow \infty$, such that $C_{n}$ has polynomial degree of accuracy $p_{n} \geqslant \tau n^{\gamma}, \tau>0,1 / 2<\gamma<1$, for each $n \in S$.

We remark that Theorem 4.1 is sharp for $s=0$, as follows from Krylov's result, and also for $s=1,2$ and 3 , as can be seen from calculations performed in [1].

Department of Computer Sciences Purdue University

Lafayette, Indiana 47907

1. L. A. ANDERSON \& W. GAUTSCHI, “Optimal weighted Chebyshev-type quadrature formulas" (To be published.)

2. S. BERNSTEIN, "Sur un système d'équations indéterminées," J. Math. Pures Appl., v. 17, 1938, pp. 179-186.

3. L. GATTESCHI, "Sulla non esistenza di certe formule di quadratura," Univ. e Politec. Torino Rend. Sem. Mat., v. 24, 1964/65, pp. 157-172. MR 32 \#4846.

4. V. I. KRYLOV, "Mechanical quadratures with equal coefficients for the integrals $\int_{0}^{\infty} e^{-x} f(x) d x$ and $\int_{-\infty}^{\infty} e^{-x^{2}} f(x) d x$," Dokl. Akad. Nauk BSSR, v. 2, 1958, pp. 187-192. (Russian) MR 22 \#861.

5. H. E. SALZER, "Equally weighted quadrature formulas over semi-infinite and infinite intervals," J. Math. Phys., v. 34, 1955, pp. 54-63. MR 16, 1055.

6. H. S. WILF, "The possibility of Tschebycheff quadrature on infinite intervals," Proc. Nat. Acad. Sci. U. S. A., v. 47, 1961, pp. 209-213. MR 23 \#A2683. 